\title{
Ocular Surface Preparation Before Keratoprosthesis Implantation
}

\author{
Katarzyna Krysik · Piotr Miklaszewski · Dariusz Dobrowolski · \\ Anita Lyssek-Boroń · Beniamin O. Grabarek (D) · Edward Wylęgała
}

Received: August 15, 2021 / Accepted: October 29, 2021 / Published online: November 22, 2021

(C) The Author(s) 2021

\begin{abstract}
Introduction: This study aimed to evaluate the surgical treatment results for conjunctival limbal autograft (CLAU) and keratolimbal allograft (KLAL) in various types of limbal stem cell deficiency (LSCD) etiologies performed in order to achieve a stable ocular surface prior to KPro implantation.

Methods: We analyzed the outcomes of the surgical treatment of 43 eyes of 39 patients with LSCD as an initial treatment preparing patients' ocular surface for KPro implantation. The most
\end{abstract}

K. Krysik · A. Lyssek-Boroń

Department of Ophthalmology, Faculty of Medicine in Zabrze, University of Technology in Katowice, 41800 Zabrze, Poland

K. Krysik · P. Miklaszewski · D. Dobrowolski ·

A. Lyssek-Boroń

Department of Ophthalmology with Paediatric

Unit, 5th Regional Hospital in Sosnowiec, 41-200

Sosnowiec, Poland

D. Dobrowolski · E. Wyleggała

Chair and Clinical Department of Ophthalmology, Division of Medical Science in Zabrze, Medical University of Silesia in Katowice, 40-760 Katowice, Poland

B. O. Grabarek $(\bowtie)$

Department of Histology, Cytophysiology and Embryology, Faculty of Medicine in Zabrze,

University of Technology in Katowice, 41-800

Zabrze, Poland

e-mail: bgrabarek7@gmail.com common causes were ocular trauma (50.7\%), mainly alkali burns (77\%); autoimmune causes, mainly ocular cicatricial pemphigoid (OCP; 17.4\%); infection (15.9\%) including Lyell's syndrome/Stevens-Johnson syndrome (LS/SJS; 16\%). In all 17 eyes operated on with CLAU, this procedure was performed once. Similarly, one uncomplicated KLAL procedure in one eye was performed in 10 women and 19 men. In another one woman and three men, KLAL was performed in both eyes. In one man with Lyell's syndrome, the KLAL operation was performed three times in one eye. Follow-up was at least 12 months.

Results: Visual acuity (VA) improved in 17 eyes (31\%) and remained unchanged in 38 eyes (69\%). VA improved from light perception to hand movements in three eyes (16\%) from the CLAU group of patients and eight eyes (15\%) from the KLAL group; VA improved from hand movements to finger counting in two eyes $(12 \%)$ post CLAU and two eyes (4\%) post KLAL operation. The most common complication of surgical treatment was persistent epithelial defect that was refractory to medical treatment in 32 eyes (58\%), 5 eyes post CLAU and 27 post KLAL. Corneal conjunctivalization (19\%) and neovascularization (29\%) were present on the corneal edge of the graft. Symblephara recurred within 3 months in nine eyes (17.3\%) after KLAL, including four eyes that had been chemically burned and five eyes with LS/SJS. 
Discussion: Pretreatment with CLAU or KLAL procedures in severely damaged ocular surfaces allows the ocular surface to be prepared for safe KPro implantation with sufficient tissue surroundings with less conjunctivalization and deeper conjunctival fornices.

Keywords: Allograft; Conjunctival limbal autograft; Corneal conjunctivalization; Keratoprosthesis; Keratolimbal neovascularization

\section{Key Summary Points}

Why carry out this study?

Restoration and maintenance of ocular surface integrity and homeostasis are the main purposes of ocular reconstructive surgery.

The purpose of this study is to report on a sample of patients who underwent surgical treatment to restore ocular surface homeostasis prior to Boston KPro type I implantation.

\section{What was learned from the study?}

Pretreatment with conjunctival limbal autograft (CLAU) or keratolimbal allograft (KLAL) procedures in severely damaged ocular surfaces allows the eye to be prepared for safe KPro implantation with sufficient tissue surroundings to fix the implant properly; additionally restoration of conjunctival fornices improve efficacy of topical treatment.

Because of the complex nature of the total limbal stem cell deficiency (LSCD), permanent inflammation and different factors leading to this state, successful corneal surface reconstruction with autografts or allografts is still a demanding and high-risk treatment.

\section{INTRODUCTION}

Restoration and maintenance of ocular surface integrity and homeostasis are the main purposes of ocular reconstructive surgery [1]. Subsequent treatment leading to improvement of visual function depends on many factors. The intact corneal epithelium covering the corneal surface is crucial for its protection and transparency, and it is essential for good visual acuity (VA). The limbus acts as a barrier, preventing the conjunctival epithelium from growing over the corneal surface $[2,3]$. Damage to the limbal stem cells (LSCs) leads to LSC deficiency (LSCD), resulting in epithelial breakdown and epithelial defects, conjunctival proliferation over the cornea with subsequent corneal opacification and vascularization, ulceration, corneal melting, and even corneal perforation in advanced tissue damage [4]. In severe cases, the fibrovascular pannus may completely cover the cornea, leading to loss of vision. The invasion of the dermal epithelium on the ocular surface may occur when injury effects the eyelid margins [5]. All disorders make reconstructive surgery highly challenging. LSCD can be caused by genetic defects, systemic immune-mediated diseases, or secondary injury or insult to the LSCs and their microenvironment, which is the cause in most cases [5].

The choice of treatment method and prognosis for successful treatment is multifactorial. Concomitant eyelid pathologies, such as lagophthalmos, lid retraction, entropion and ectropion, symblepharon, fornix shortening, trichiasis and lid margin keratinization, damage of the meibomian and lacrimal glands, dry eye, ocular inflammation and infection, and uncontrolled systemic disorders, first require reconstruction of an appropriate limbal microenvironment. This involves limiting and controlling inflammation, improving the tear film, and promoting the differentiation of the corneal epithelial cells with medical and surgical methods. Appropriate management of adnexal pathology prior to ocular surface reconstruction is necessary $[4,6,7]$.

Successful ocular surface reconstruction requires a systematic and stepwise approach. 
Corneal surgery must be postponed until an inflammation-free period is reached and lid and adnexal abnormalities have been corrected [7]. Unilateral LSCD, in most cases resulting from ocular trauma-mainly burns-can be partial or total. In cases where vision is affected, surgical options include sequential removal of the conjunctival epithelium from the corneal surface and removal of fibrovascular pannus with amniotic membrane application [8]. Cell-based therapy, in the form of direct or cultivated autologous LSCs, provides long-term stabilization of the ocular surface with significant improvement in VA $[8,9]$.

Bilateral LSCD is more frequently a result of systemic or autoimmune conditions, mainly ocular cicatricial pemphigoid (OCP), Stevens-Johnson syndrome (SJS), also known as the Lyell's syndrome, where there is no autologous source of healthy donor limbal tissue. Surgical management of bilateral LSCD is based on the two following approaches: cell-based therapies, including allogenic limbal sources (cultivated limbal epithelial transplantation (CLET) or autologous cultivated oral mucosal epithelial transplantation (COMET), amniotic membrane transplantations, keratolimbal allograft (KLAL) and keratoprostheses (KPros) or synthetic corneas $[2,6-8,10-16]$.

The study aimed was to evaluate the surgical treatment results for CLAU and KLAL in various types of limbal stem cell deficiency etiologies performed in order to achieve a stable ocular surface prior to KPro implantation.

\section{METHODS}

We retrospectively reviewed the outcomes of the surgical treatment of 43 eyes of 39 patients with total LSCD. All surgeries were performed between June 1, 2018 and January 31, 2020. Patients were recruited from the Ophthalmology Department of Saint Barbara Hospital, Trauma Centre, Sosnowiec, Poland. Data from the medical records included demographics, medical history, preoperative and postoperative best spectacle-corrected visual acuity (BSCVA) measured using the Snellen VA chart, outcomes and complications of surgery, results of accessory examinations (microbial tests), postoperative intraocular pressure (IOP), graft rejection, and other comorbidities and complications.

The study was conducted according to the guidelines of the Declaration of Helsinki. Because this study was a retrospective analysis of data obtained in clinical routine care at an academic university setting, consent of the ethics committee was not required. According to the Polish statute, this is a non-interventional study (Article 37a (1) of the Pharmaceutical Law) and therefore, on the understanding of the Act of 5 December 1996 on the professions of doctor and dentist, does not require the opinion of the bioethical committee and does not constitute a clinical trial. Informed consent was obtained from all subjects involved in the study.

Indications for surgical treatment were chemical or thermal burns and cicatrizing conjunctival disease, such as OCP, SJS/LS. The diagnosis of total LSCD was made clinically on the basis of the medical history, patient examination, and response to previous treatment. All patients suffered from severe ocular surface pathology with total LSCD and underwent multistage, repetitive surgical treatment. Additional simultaneous intensive local and general immunosuppressive and anti-inflammatory medications were applied. Total LSCD clinical manifestations comprised severe limbal ischemia of four quadrants, persistent limbal inflammation, non-healing persistent corneal epithelial defects often leading to ulcerations, corneal melting or perforation, corneal superficial and deep neovascularization, fibrovascular pannus, scarring, keratinization and calcification of the corneal surface. Ocular surface and adnexal involvement did not allow direct application of Boston KPro type I. Additional treatment was necessary to improve the local conditions on the surface.

All patients with diagnosed total LSCD were treated using two different surgical methodsKLAL and conjunctival limbal autograft (CLAU)—with the purpose of achieving a stable ocular surface prior to KPro implantation. In some eyes, additional amniotic membrane transplantation (AMT) was performed, serving 
as accessory tissue covering the reconstructed fornix or in conjunction with a stem cell procedure; this contributed a substrate for stem cell proliferation and epithelial migration, accelerating re-epithelialization following surgery. AMT was applied routinely as a first-line treatment after ocular burns to suppress inflammation, facilitate epithelialization, and prevent cicatricial complications in acute chemical and thermal burns. Application of the amniotic membrane was performed immediately after admission to the ophthalmic emergency center. Only eyes with tear film secretion qualified for the treatment.

In the patients, the severe ocular surface pathology also demanded medical and frequently surgical treatment of adnexal pathologies, such as ectropion, entropion, trichiasis, dacryocystitis, symblepharolysis, ankyloblepharolysis, and other lid procedures. During KLAL surgery and frequently one-time restoration of lost conjunctival fornixes, the AMT on the bare sclera in the bottom of the newly created fornix was necessary. The monolayer amniotic patch was sutured epithelial-side down using 10-0 nylon running or interrupted sutures extending to the edges of the palpebral conjunctiva and the external margin of the KLAL graft.

The KLAL technique involved preparation of the donor tissue and $360^{\circ}$ corneoscleral rings remaining after corneal trephination for penetrating keratoplasty. The limbal tissue attached to a corneal carrier was harvested from the cadaveric eyes and transplanted to the recipient eye. Oversized donor grafts with $2-3 \mathrm{~mm}$ scleral rims were preserved in a cold storage medium of Eusol-C solution (Alchimia, S.r.I., Ponte S. Nicolo, Italy). During surgery, the posterior onehalf to two-thirds of stromal and scleral tissues were removed by lamellar dissection using a sharp, rounded steel crescent blade with the freehand technique. In areas of symblepharon, conjunctival tissue was first recessed at the limbus and then undermined to allow the conjunctival tissue to fall back. A $360^{\circ}$ conjunctival peritomy was carried out and the conjunctiva was resected to $2.0-3.0 \mathrm{~mm}$ from the limbus to expose an adequately sized bed of denuded sclera on which to position the KLAL tissue. All corneal pannus and corneal irregularities were smoothed and removed with a rounded blade. The keratolimbal donor allograft was positioned and sutured with 7-0 Vicryl sutures to the recipient scleral bed. The corneal part of the allograft usually fitted closely to the recipient cornea. The recipient's primary pushed-back conjunctiva was sutured tightly with 10-0 Vicryl sutures just outside the limbus. All surgical procedures were performed under general anesthesia. Patients were hospitalized for 1-2 days after KLAL and followed up 2 weeks after hospitalization and monthly for 6 months.

CLAU is a procedure in which limbal tissue attached to a conjunctival carrier is transplanted from the healthy eye to the contralateral stem cell-deficient eye [17]. The main advantage over the allograft procedure is that there is no need for general immunosuppression. This technique was applied in unilateral LSCD. In the recipient eye, conjunctival peritomy was performed in the region of consecutive autografts. The superficial keratectomy was performed to remove the abnormal epithelium and fibrovascular epithelium. The donor eye tissue was then carefully harvested from the healthy eye. The tissue source was superior and inferior limbus of donor eye. Two trapezoidshaped limbal grafts, measuring $5.0 \mathrm{~mm}$ at the limbus and extending $4.0-6.0 \mathrm{~mm}$ posterior to the limbus, were demarcated with a rounded blade. The thickness of the limbal tissue do not extend $150 \mu \mathrm{m}$. The corneal margin of the autograft was extended through the palisades of Vogt to ensure isolation of stem cells. After careful dissection of the graft margins, it was transferred to the donor eye with proper epithelial and limbal orientation. The graft was then sutured with 10-0 Vicryl sutures to the recipient eye scleral bed. To avoid stem cell damage, no sutures were placed through the limbal or corneal margins. The donor eye conjunctiva was sewn back with 10-0 nylon sutures. All procedures were performed under local anesthesia, with the use of drops of $0.5 \%$ proxymetacaine and subconjunctival injection of $2 \%$ xylocaine for easier separation of the conjunctiva from Tenon's layer if necessary. Patients were hospitalized for 1 day and 
followed up 2 weeks after the procedure and monthly for 6 months.

Postoperative treatment consists of 14 days of topical fluoroquinolone administered five times a day and dexamethasone therapy with the following regimen: seven times a day for 14 days, five times a day for 14 days, three times a day for 30 days, and once a day afterwards. In the KLAL group prednisone was taken orally at a dose of $20 \mathrm{mg}$ for 14 days and then $10 \mathrm{mg}$ for the next 14 days. General immunosuppressive treatment included mycofenolate mofetil $500 \mathrm{mg}$ BID applied in long-term administration.

After both KLAL and CLAU surgery, intensive management of epithelialization and local and systemic immunosuppression, control of inflammation, and management of coexisting glaucoma and other comorbidities and complications were conducted. A minimum of 1 year after ocular surface reconstruction, Boston KPro type I was implanted.

\section{RESULTS}

Between June 1, 2018 and January 1, 2020, 69 surgeries preparing the ocular surface for KPro implantation were performed. Follow-up was at least 12 months. These surgeries involved 22 procedures in a group of 17 women (18 eyes), where the mean age was $54.32 \pm 16.5$ (range $45-78$ years), and 47 procedures in a group of 34 men (37 eyes), where the mean age was $54.37 \pm 15.14$ (range $28-90$ years).

In 6 eyes of 6 women and 11 eyes of 11 men, an uncomplicated CLAU procedure was performed once. Similarly, one uncomplicated KLAL procedure in one eye was performed in each of 10 women and 19 men. In another one woman and three men, KLAL was performed in both eyes. In one man with Lyell's syndrome, the KLAL operation was performed three times in one eye. Because of severe scarring of the ocular surface, despite prior KLAL surgery, two women and five men (two men post KLAL operation in both eyes) required KLAL to be reperformed, and two women and three men who underwent CLAU were operated on with the KLAL technique.
In all 17 eyes operated on with CLAU, we used this technique once. In one eye of a woman with OCP and two eyes of one male patient with Lyell's syndrome, after KLAL, corneal perforation was recognized, and penetrating keratoplasty (PK) à chaud was performed. Healthy eyes did not reveal any LSCD signs in follow-up.

The time between the ocular pathology report and ocular surface reconstruction was $29 \pm 6$ months (from 12 to 54 months). The oldest patient, a 90-year-old man, lost useful VA in both eyes and suffered from LSCD complications after a misfire explosion in 1949.

The main ocular surface pathologies requiring prior surgical preparation before KPro implantation were reported. The most common causes were ocular trauma (50.7\%), mainly alkali burns (77\%); autoimmune causes, mainly OCP (17.4\%) including LS/ SJS (16\%) and infection (15.9\%). The indications and details of the surgical treatment of the ocular surface pathology before KPro implantation are presented in Table 1.

Because of the autologous origin of the graft, after CLAU, we used only topical medication, with no necessity for general immunosuppression. Patients received topical dexamethasone $(0.1 \%)$ and broad-spectrum antibiotic (fluoroquinolones; moxifloxacin or levofloxacin) eye drops with regimen described before. Continuous lubrication with artificial tears eye drops was highly important. KLAL patients were additionally treated with oral steroid for 1 month and with oral immunosuppressive agents. Postoperative medical treatment after KLAL included a topical steroid (dexamethasone seven times a day), broad-spectrum antibiotic (fluoroquinolones; moxifloxacin or levofloxacin), aminoglycosides (gentamycin), and strong systemic immunosuppression, including steroids, cyclosporine A, azathioprine, and mycophenolate mofetil. It was crucial to ensure persistent intensive lubrication to support favorable conditions for epithelialization and to maintain successful surgical and medical treatment. Topical antimicrobial therapy was routinely applied for 21 days and extended if needed. Steroid doses were tapered (one drop less each month) to maintenance 
Table 1 Surgical techniques and indications for surgery in ocular surface pathology

\begin{tabular}{llll}
\hline Characteristics & $\begin{array}{l}\text { Total }(\boldsymbol{N}=\mathbf{6 9}) \\
\boldsymbol{N}(\%)\end{array}$ & $\begin{array}{l}\text { Women }(\boldsymbol{n}=\mathbf{2 2}) \\
\boldsymbol{N}(\%)\end{array}$ & $\begin{array}{l}\text { Male }(\boldsymbol{n}=\mathbf{4 7}) \\
\boldsymbol{N}(\%)\end{array}$ \\
\hline CLAU & $17(24.6)$ & $6(32.0)$ & $11(68.0)$ \\
Indication for surgery & & & $8(17.0)$ \\
Ocular trauma & $12(17.4)$ & $4(18.2)$ & - \\
Autoimmune causes & - & - & $3(6.4)$ \\
Infection & $5(7.2)$ & $2(9.1)$ & - \\
LS/SJS & - & - & $36(52.0)$ \\
KLAL & $52(75.4)$ & $16(48.0)$ & $17(36.2)$ \\
Indication for surgery & & & $7(14.9)$ \\
Ocular trauma & $23(33.3)$ & $6(27.3)$ & $5(10.6)$ \\
Autoimmune causes & $12(17.4)$ & $5(22.7)$ & $7(14.9)$ \\
Infection & $6(8.7)$ & $1(4.5)$ & $4(18.2)$ \\
LS/SJS & $11(16.0)$ & &
\end{tabular}

treatment five times a day. Modifications were applied according to the progress of the disease.

To create suitable conditions for KPro implantation in seven eyes (13\%), KLAL was repeated, and in one eye, it was performed three times. In eight eyes treated with the KLAL technique, AMT was used to cover the restored fornixes.

The most common complication of surgical treatment was persistent epithelial defect that was refractory to medical treatment. This complication occurred in 32 eyes (58\%), 5 eyes after CLAU, and 27 after KLAL, mainly in patients with Lyell's syndrome or autoimmunological conditions. In addition, abnormal tear production dominated in these groups of patients. The purpose of intensive medical treatment was to increase the ocular surface moisture with preservative-free lubricants, a $20 \%$ solution of autologous serum drops, and cyclosporine $\mathrm{A}$ drops, as well as to limit existing tear evaporation with bandage soft contact lenses, partial tarsorrhaphy, ptosis with botulin toxin, or a constant eye patch. As an adjunctive therapy, anti-collagenases, such as orally administered doxycycline (100 mg twice daily for 6-8 weeks) and vitamin $\mathrm{C}$ to facilitate collagen synthesis, were applied.
Corneal conjunctivalization (19\%) and neovascularization (29\%) were present on the corneal edge of the graft. Symblepharon recurred within 3 months in nine eyes (17.3\%) after KLAL, including four eyes that had been chemically burned and five eyes with LS/SJS. They were dissected during separate surgery or subsequent KLAL.

Glaucoma, which is frequently associated with severe ocular surface disease resulting from injuries, mainly alkali burns, multiple surgical procedures, and long-term use of topical corticosteroids, was managed aggressively in patients undergoing limbal transplantation. A simple way to monitor IOP elevation was regular eye globe axial length measurements.

Glaucoma or ocular hypertension was reported in 28 eyes (51\%) and is crucial to ensure the best prognosis for further visual rehabilitation. Eight eyes (14.5\%) required surgical intervention to normalize IOP that was refractory to medical treatment, including three trabeculectomies, three transscleral cyclophotocoagulation procedures, and two Ex-Press glaucoma-shunt implantations.

Because of the complex and hard nature of underlying conditions leading to corneal surface disintegrity, the only goal of our treatment 
was to achieve preparation of the ocular surface for KPro implantation, not to improve VA. However, the postoperative VA in the study group of patients was improved in 17 eyes (31\%) and remained unchanged in 38 eyes (69\%). VA was improved from light perception to hand movements in three eyes (16\%) from the CLAU group of patients and eight eyes $(15 \%)$ from the KLAL group, as well as from hand movements to finger counting in two eyes (12\%) after CLAU and two eyes (4\%) after KLAL operation. VA improvement resulted from a combination of various factors, which were as follows: slight partial corneal neovascularization disappearance, more stable and complete tear film, partial corneal epithelium regeneration, and a limitation of corneal surface inflammation.

Boston type I keratoprosthesis obtained approval for reimbursement from public finances in Poland in July 2019. Since then, keratoprostheses have been implanted in a group of patients after the KLAU and CLAU initial procedures. At the date of preparation of the manuscript, 12 patients had a follow-up period of at least 6 months after the implantation of the keratopthesis. Nine eyes were operated on after KLAL, and three after CLAU. Ocular surface disorders after implantation were observed only in two patients after KLAL, the surgical procedure used for both cases was conjunctival flap surgery with the displacement of the conjunctival tissue over corneal necrosis in the area of the keratoprosthesis interface. Both interventions were successful.

Visual acuity after keratoprosthesis after KLAL procedure resulted in BCVA above 0.5 (0.9-0.5) only in three cases, the remaining two persons maintained visual acuity of $0.2-0.3$, and four others below 0.1. The reason for the low visual acuity was glaucoma in four cases and macular RPE atrophy in two eyes.

Figure 1 presents an eye before KLAL procedure for LSCD due to Lyell's syndrome. Figure 2 shows an eye after KLAL procedure for LSCD due to Lyell's syndrome. Figure 3 shows an example of an eye after KLAL and KPro procedure for LSCD due to Lyell's syndrome.

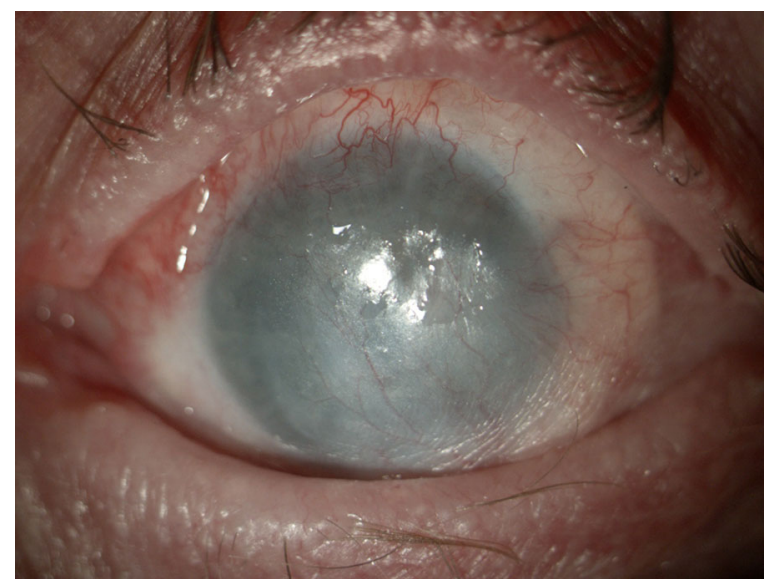

Fig. 1 Eye before KLAL procedure for LSCD due to Lyell's syndrome

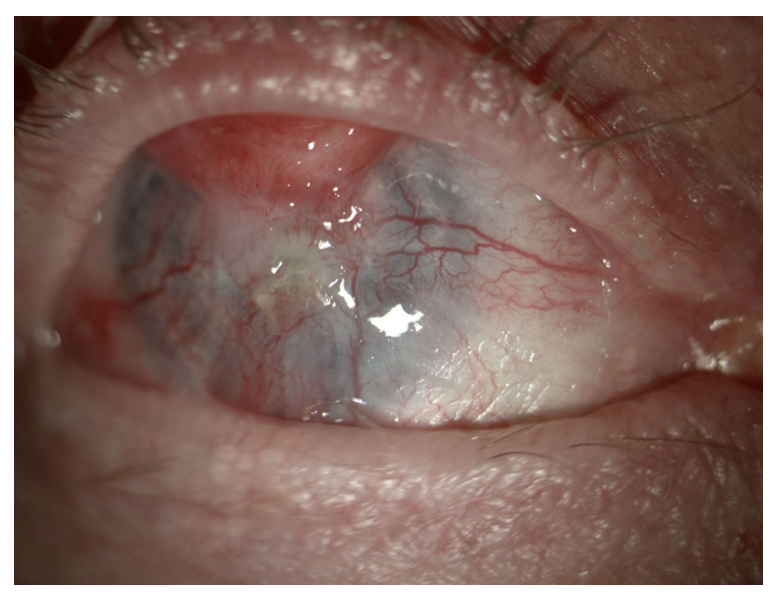

Fig. 2 Eye after KLAL procedure for LSCD due to Lyell's syndrome

\section{DISCUSSION}

Diseases affecting the cornea are a major cause of blindness worldwide. Globally, corneal opacity of various origins is the fourth-leading cause of bilateral blindness, following cataract, glaucoma, and age-related macular degeneration [10]. Trauma, inflammation, and congenital diseases are the main causes of LSCD, frequently leading to irreversible corneal destruction. Prior to any attempt at LSC transplantation, the ocular surface must be optimized. Except for a proper surgical approach, the following are the two most important 


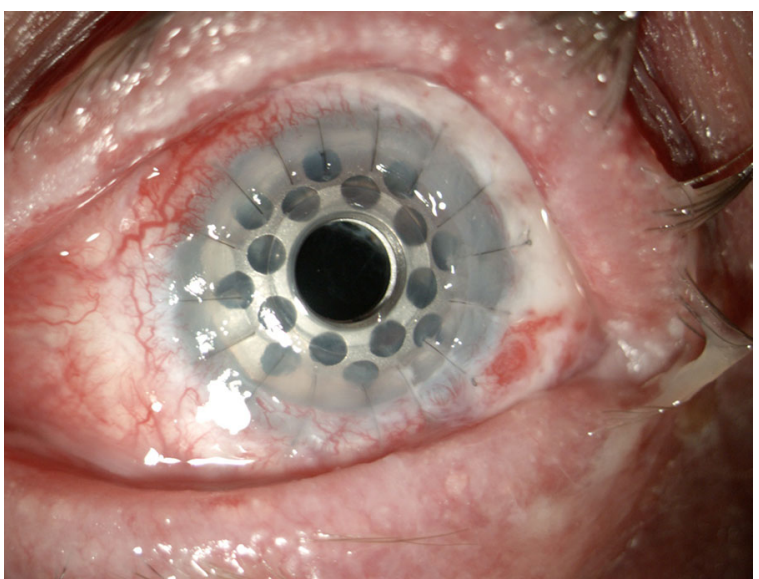

Fig. 3 Eye after KLAL and KPro procedure for LSCD due to Lyell's syndrome

general principles: controlling causative factors and controlling comorbid conditions [6].

KPros offer a therapeutic alternative to patients with corneal blindness who cannot undergo successful corneal allograft [10]. However, to implant a prosthesis, it is necessary to restore and stabilize the ocular surface.

In the current study, we reported that the therapeutic approach to ocular surface reconstruction in LSCD before KPro implantation is challenging and unpredictable. This results from the extent of involvement of the limbus (partial or total), involvement of surrounding tissues, and unilaterality or bilaterality of LSCD [6-8]. Often, a repeated surgical approach is necessary to achieve a stable ocular surface.

The transplantation procedures presented in our report are usually more favorable than local or general medication using adhesive tissues or other forms of treatment. In LSCD, this is frequently the only possible approach to restoring the ocular surface integrity. An important aspect of surgical treatment for LSCD is the availability of tissue necessary for the procedure [18].

With the KLAL technique, large numbers of stem cells can be transplanted. KLAL permits the treatment of LSCD eyes when there is no available living-related or autograft tissue [18]. This technique is performed to treat severe bilateral ocular surface disorders secondary to LSCD. In our study, the overall success of KLAL depended on the causes of LSCD. It was consistent with clinical reports on KLAL results in LS/SJS and autoimmunological patients $[7,18]$, where the complexity of autoimmunological processes leading to KLAL failure demanded strong immunosuppression, frequently with two different drugs and systemic and topical steroids. We used cyclosporine A or azathioprine and mycophenolate mofetil.

Liang et al. [19] reported that successive KLAL was performed after failure of the first KLAL in 11 of 39 study group patients; nine eyes demanded a second, one eye a third, and another one eye a fourth KLAL procedure. In our study, only five eyes demanded re-KLAL, and one eye was operated on three times with this technique. The prognosis for successful KLAL survival is related to persistent inflammation, severe dry eye, or asymptomatic and progressive rejection of KLAL. Thus, the higher survivability for KLAL in our study probably resulted from selected indications for ocular surface reconstruction, mainly post ocular trauma.

Many reports have suggested that some risk factors for KLAL failure include chronic inflammation, dry eye, symblepharon, keratinization, lid abnormalities, and increased IOP [7]. Glaucoma or increased IOP may be a comorbidity secondary to the underlying LSCD, and the use of oral and topical steroids may lead to the development of increased IOP. This state often demands drainage device placement or medical antiglaucomatous therapy [7, 13, 18]. Glaucoma or ocular hypertension was reported in 28 eyes (51\%). Most of these eyes were treated with one or three topical agents (timolol, brimonidine, brinzolamide) or referred to trabeculectomy with peripheral iridectomy in three eyes, transscleral cyclophotocoagulation in three eyes, and Ex-Press glaucoma-shunt implantation in two eyes.

In patients with unilateral LSCD, KLAL is a surgical alternative for those who fear damage to the healthy fellow eye if it is used as a source of limbal stem cells. CLAU is particularly effective in treating patients with conjunctival scarring and inflammation. In addition to limbal tissue, conjunctival tissue is also transplanted. This is the method of choice for unilateral LSCD 
$[11,20]$. It is thought that harvesting about $40 \%$ of stem cells would not destabilize the donor eye [21]. Long-term follow-ups have shown a good graft survival rate [3]. In our study, five eyes $(29 \%)$ required reoperation. In the case of corneal surface instability and subsequent scarring, delayed epithelial healing requiring AMT, and progressive conjunctival ingrowth overriding the grafts, we decided not to repeat CLAU operations.

The VA improvement after CLAU depends on the graft size and drops with smaller grafts [21]. The study aimed was to evaluate the surgical treatment results for CLAU and KLAL in various types of limbal stem cell deficiency etiologies performed in order to achieve a stable ocular surface prior to KPro implantation. A stable ocular surface was achieved in $71 \%$ of cases. This result remains consistent with Ozer et al.'s [1] study, where CLAU transplantation was applied to the patients at least 3 months after injury, and normal epithelialization was achieved in $68 \%$ of eyes. Epithelialization failed in only four eyes (13\%) during the postoperative period, three of which had severe recurrent cicatricial eyelid disorders despite reconstruction.

The rate of postoperative glaucoma after CLAU ranges from $6 \%$ to $32 \%$, increasing with subsequent optical allografting $[1,18,20]$. The reason for glaucoma development in CLAU eyes was interpreted as the consequence of alkali injury-induced trabecular meshwork damage. In our report, glaucoma or increased IOP was observed in four eyes (23.5\%), requiring topical medication in two eyes and surgical intervention in the other two eyes, with one trabeculectomy with peripheral iridectomy and one Ex-Press glaucoma-shunt implantation. The other explanation for a lower frequency of increased IOP after CLAU than after KLAL is the lack of need to use steroids in general medication and smaller doses of topical steroids.

Our observations indicate that waiting at least 1 year from reconstructive surgery of the ocular surface before the optical operationKPro implantation in our study group-fosters better final outcomes. Of course, the success of KPro does not solely depend on the limbal niche restoration but on other factors such as lid malformations, dry eye, and protracted inflammation. Ozer et al. [1] presented a similar view in relation to CLAU and penetrating keratoplasty in eyes with LSCD. Similarly, Yao et al. [9] suggested that there should be a minimum of 12 months between CLAU and associated deep anterior lamellar keratoplasty procedures. We presume that the failures of limbal autoand allografts in our study mostly occurred because of advanced-stage ocular surface destruction.

According to Özdemir et al. [22], limbal autografts seem to be superior to limbal allografting under similar ocular conditions. Perhaps the main aim of limbal allografting should be to relieve patients' symptoms first, and thereafter to prepare the corneal surface for a successful future keratoplasty-KPro implantation in our material.

The two-step surgical approach, including first KLAL or CLAU and staged KPro implantation, seems to be more effective in improving vision and maintaining long-term ocular surface stability in patients with severe ocular surface disease and LSCD. It offers a more stable ocular surface than a simultaneous procedure, along with a better prognosis of graft survival.

A limitation of this study is its retrospective nature. For this reason, in the next stages it would be reasonable to extend the analyses to the observations from prospective studies and to include the control group. Of course, it is always reasonable to increase the analyzed groups, as well as to extend the period of observation. Nevertheless, the results presented in this study are significant.

\section{CONCLUSION}

Because of the complex nature of the total LSCD and different factors leading to this state, successful corneal surface reconstruction with autografts or allografts is still a demanding and high-risk treatment. The improvement of VA, the main goal of ophthalmic treatment, falls into second place. Further investigations and development of new surgical techniques and appropriate immunosuppressive approaches are 
still necessary to improve the final outcomes of treatment.

\section{ACKNOWLEDGEMENTS}

Funding. No funding or sponsorship was received for this study or publication of this article. The authors funded the journal's rapid service fee.

Authorship. All named authors meet the International Committee of Medical Journal Editors (ICMJE) criteria for authorship for this article, take responsibility for the integrity of the work as a whole, and have given their approval for this version to be published.

Author Contributions. Conceptualization, K.K. and P.M.; methodology, K.K.; formal analysis, D.B., A.L-B.; resources, B.O.G.; data curation, K.K.; writing-original draft preparation, P.M., D.D., and A.L.-B.; writing-review and editing, K.K. and E.W.; supervision, E.W.; project administration, B.O.G. All authors have read and agreed to the published version of the manuscript.

Disclosures. Katarzyna Krysik, Piotr Miklaszewski, Dariusz Dobrowolski, Anita LyssekBoroń, Beniamin Oskar Grabarek, and Edward Wylegała have nothing to disclose.

Compliance with Ethics Guidelines. The study was conducted according to the guidelines of the Declaration of Helsinki. Because this study was a retrospective analysis of data obtained in clinical routine care at an academic university setting, consent of the ethics committee was not required. According to the Polish statute, this is a non-interventional study (Article 37a (1) of the Pharmaceutical Law) and therefore, on the understanding of the Act of 5 December 1996 on the professions of doctor and dentist, does not require the opinion of the bioethical committee and does not constitute a clinical trial. Informed consent was obtained from all subjects involved in the study.
Data Availability. All data generated or analyzed during this study are included in this published article.

Open Access. This article is licensed under a Creative Commons Attribution-NonCommercial 4.0 International License, which permits any non-commercial use, sharing, adaptation, distribution and reproduction in any medium or format, as long as you give appropriate credit to the original author(s) and the source, provide a link to the Creative Commons licence, and indicate if changes were made. The images or other third party material in this article are included in the article's Creative Commons licence, unless indicated otherwise in a credit line to the material. If material is not included in the article's Creative Commons licence and your intended use is not permitted by statutory regulation or exceeds the permitted use, you will need to obtain permission directly from the copyright holder. To view a copy of this licence, visit http://creativecommons.org/licenses/by$\mathrm{nc} / 4.0 /$.

\section{REFERENCES}

1. Ozer MD, Altinkurt E, Alparslan N. The long-term surgical outcomes of conjunctival-limbal autograft procedure with or without penetrating keratoplasty in eyes with unilateral limbal stem cell deficiency. Taiwan J Ophthalmol. 2020;10:22-8.

2. Vazirani J, Basu S, Kenia H, et al. Unilateral partial limbal stem cell deficiency: contralateral versus ipsilateral autologous cultivated limbal epithelial transplantation. Am J Ophthalmol. 2014;157: 584-90.

3. Yin J, Jurkunas U. Limbal stem cell transplantation and complications. Semin Ophthalmol. 2018;33: 134-41.

4. Wang J, Qi X, Dong Y, et al. Comparison of the efficacy of different cell sources for transplantation in total limbal stem cell deficiency. Graefes Arch Clin Exp Ophthalmol. 2019;257:1253-63.

5. Deng SX, Kruse F, Gomez JAP, et al. Global consensus on the man-agement of limbal stem cell deficiency. Cornea. 2020;39:1291-302. 
6. Atallah MR, Palioura S, Perez VL, Amescua G. Limbal stem cell transplantation: current perspectives. Clin Ophthalmol. 2016;10:593-602.

7. Han ES, Wee WR, Lee JH, Kim MK. Long-term outcome and prognostic factor analysis for keratolimbal allografts. Graefes Arch Clin Exp Ophthalmol. 2011;249:1697-704.

8. Vazirani J, Mariappan I, Ramamurthy S, Fatima S, Basu S, Sangwan VS. Surgical management of bilateral limbal stem cell deficiency. Ocul Surf. 2016;14:350-64.

9. Yao Y-F, Zhang B, Zhou P, Jiang J-K. Autologous limbal grafting combined with deep lamellar keratoplasty in unilateral eye with severe chemical or thermal burn at late stage. Ophthalmology. 2002;109:2011-7.

10. Chen M, Akpek EK, Ahmad S. Artificial corneas versus donor corneas for repeat corneal transplants. Cohrane Database Syst Rev. 2020;2020:CD009561.

11. Cheung AY, Sarnicola E, Holland EJ. Long-term ocular surface stability in conjunctival limbal autograft donor eyes. Cornea. 2017;36:1031-5.

12. Kenyon KR, Tseng SCG. Limbal autograft transplantation for ocular surface disorders. Ophthalmology. 1989;96:709-23.

13. Shanbhag SS, Saeed HN, Paschalis EI, Chodosh J. Keratolimbal allograft for limbal stem cell deficiency after severe corneal chemical injury: a systematic review. $\mathrm{Br} \mathrm{J}$ Ophthalmol. 2018;102: 1114-21.

14. Zarei-Ghanavati M, Liu C. Keratoprosthesis: current choices and future development. Graefes Arch Clin Exp Ophthalmol. 2019;8:429-31.
15. Kang JJ, de la Cruz J, Soledad-Cortina M. Visual outcomes of boston keratoprosthesis implantation as the primary penetrating corneal procedure. Cornea. 2012;31:1436-40.

16. Sayegh RR, Diaz LA, Vargas-Martin F, Webb RH, Dohlman $\mathrm{CH}$, Peli E. Optical functional properties of the boston keratoprosthesis. Invest Ophthalmol Vis Sci. 2010;51:857-63.

17. Moshirfar M, Thomson AC, Ronquillo Y. Limbal epithelial transplant. Treasure Island: StatPearls; 2021.

18. Cheung AY, Holland EJ. Keratolimbal allograft. Curr Opin Ophthalmol. 2017;28:377-81.

19. Liang L, Sheha H, Tseng SC. Long-term outcome of keratolimbal allograft with or without penetrating keratoplasty for total limbal stem cell deficiency. JAMA Ophthalmol. 2009;127:1428-34.

20. Shimazaki J, Shimmura S, Tsubota K. Donor source affects the outcome of ocular surface reconstruction in chemical or thermal burns of the cornea. Ophthalmology. 2004;111:38-44.

21. Liang L, Sheba H, Li J, Tseng SC. Limbal stem cell transplantation: new progresses and challenges. Eye. 2009;23:1946-53.

22. Özdemir Ö, Tekeli O, Örmek K, Arslanpençe A, Yalçindağ NF. Limbal autograft and allograft transplantations in patients with corneal burns. Eye. 2004;18:241-8. 